\title{
TESTING OF MAGNETIC COMPASSES
}

\author{
By R. L. Sanford
}

\section{ABSTRACT}

The work of the Bureau of Standards on magnetic compasses was undertaken in response to requests for information and cooperation from the War Department and the United States Shipping Board. It consisted of studies of the behavior of various types of compasses, the construction of special apparatus for testing purposes, and special investigations. The information thus obtained was utilized in the preparation of specifications for various types of compasses and also in the preparation of specifications for testing.

In the course of this investigation certain facts concerning the general characteristics of compasses were brought out which were considered to be of interest to users of magnetic compasses. This paper gives a brief discussion of the principal performance characteristics of magnetic compasses together with a description of some of the apparatus which is used at the Bureau for testing.

\section{CONTENTS}

Introduction.

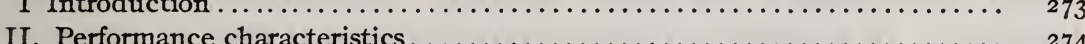

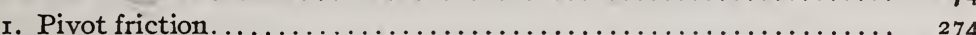

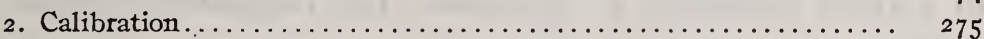

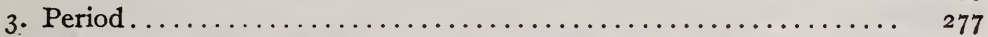

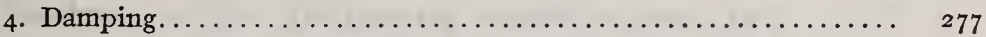

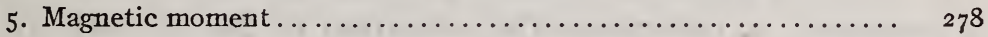

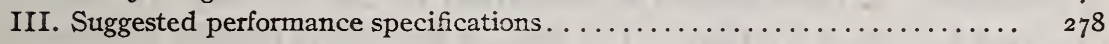

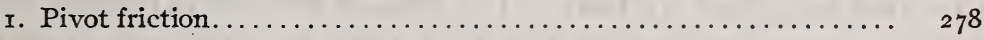

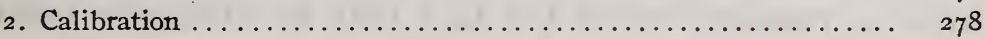

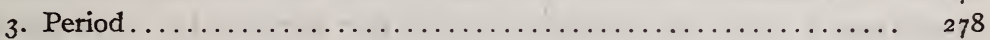

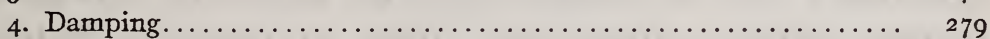

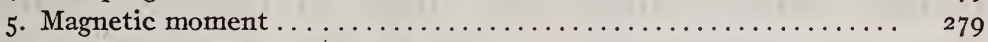

IV. Summary . . . . . . .

\section{INTRODUCTION}

During the early part of I 917 the Bureau of Standards was called upon by the War Department and the U. S. Shipping Board for cooperation in connection with the preparation of specifications for magnetic compasses and the development of methods for their testing. - As this was a subject which had not previously been taken up by the Bureau and as there was little information available on the subject, a considerable amount of experimental work has been done, involving not only a study of the behavior of a $181068^{\circ}-20$ 
Scientific Papers of the Bureau of Standards, Vol. 16

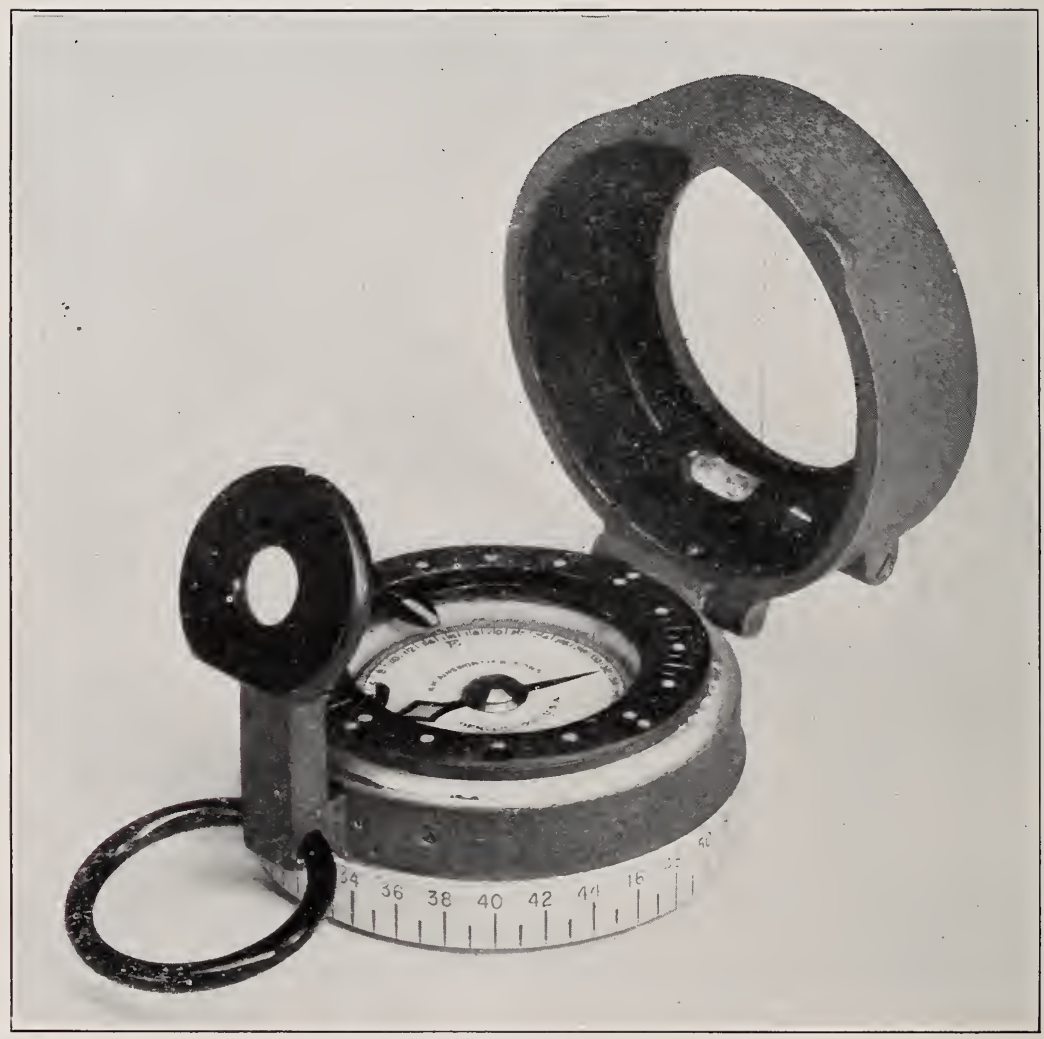

FIG. I.-Lensatic compass 
number of different types of instruments but also more detailed studies of individual features of construction. While this study of compasses was by no means comprehensive, certain general facts regarding their performance and testing were brought out which would seem to be of interest not only to purchasers of compasses in large quantities, but also to individual users.

Most of the factors which determine the accuracy and behavior of a magnetic compass are affected not only by its design, but also by the quality of materials and workmanship which enter into its construction. The suitability of a given type of instrument for a particular class of service is governed by its design, but individual instruments generally vary somewhat in accuracy and performance. In this paper it is proposed to consider briefly the factors governing the behavior of individual instruments rather than the design of compasses suitable for various purposes.

\section{PERFORMANCE CHARACTERISTICS}

The performance characteristics which can be easily determined by test and which may be taken as criteria of the quality of individual instruments are pivot friction, calibration, period, and damping. In addition to these characteristics it may be desirable in certain instances to determine the magnetic moment of the needles.

I. PiVot Friction.-Before proceeding with a compass test it is necessary to determine the condition of the bearing by means of a test for pivot friction. The reason for this is readily apparent from a consideration of the fact that an otherwise satisfactory instrument may be rendered unfit for service by excessive pivot friction resulting either from injury, or imperfect materials or from poor workmanship. Pivot friction, or rather the degree of freedom from it, is sometimes termed "sensibility," as its effect is to make a compass insensitive to slight changes of direction. This makes the reading of a compass uncertain by an amount which has been called by one writer ${ }^{1}$ the "angle of uncertainty.." The usual method of test is very simple and requires no auxiliary apparatus other than a small permanent magnet or, better, a small coil. It consists of deflecting the compass by a small specified angle and noting the difference in reading before and after the deflection. The test is repeated, deflecting the compass in the opposite direction and also with the compass bowl oriented in 
a number of different directions, usually with the lubber line initially opposite each of the cardinal points. It is difficult to repeat results exactly, but there is generally no difficulty in differentiating between a satisfactory and an unsatisfactory instrument.

2. Calibration.-The term calibration includes all of the factors other than pivot friction which affect the accuracy of reading of a compass. The principal causes of error are: $(a)$ Incorrect orientation of the magnet needles on the card; $(b)$ incorrect graduation of the card or scale; $(c)$ eccentricity of mounting; $(d)$ the presence of magnetic materials; $(e)$ incorrect adjustment of sighting devices (lubber line, prisms, slits, etc.).

In mounting the magnet needles on a compass card it is essential that they be so placed that the resultant magnetic axis is parallel to the direction of a line through the north and south points. If this condition is not fulfilled, there will be a corresponding error in the reading which will be the same in magnitude and direction for all headings.

Errors due to incorrect graduation of the card or scale are usually small in high-grade instruments. Very poor graduations can generally be detected by inspection. Such errors may be in either direction and vary in magnitude.

If the axis of support of the card does not pass through the center of graduation, errors are introduced which vary from zero to a maximum, depending upon the heading on which a reading is taken.

If magnetic materials are present, the needles may be deviated from their proper position. These effects will vary in magnitude and sign, according to the relative positions of magnetic impurities and the magnet needles.

The principal requirement for sighting devices for compassessuch as prisms, slits, etc.-is that the line of sight shall always lie in the vertical plane passing through the center of suspension. If this condition is not fulfilled, the result is similar to that of eccentricity in the mounting of the card itself.

Except in the case of a manufacturer who is developing a new instrument, it is generally not necessary to analyze the calibration errors into their component parts, and a simple test is all that is required.

A simple testing stand (shown in Fig. 2) was built at the Bureau for the purpose of determining the calibration error of compasses. It consists of a table which can be rotated about 
Scientific Papers of the Bureau of Standards, Vol. 16

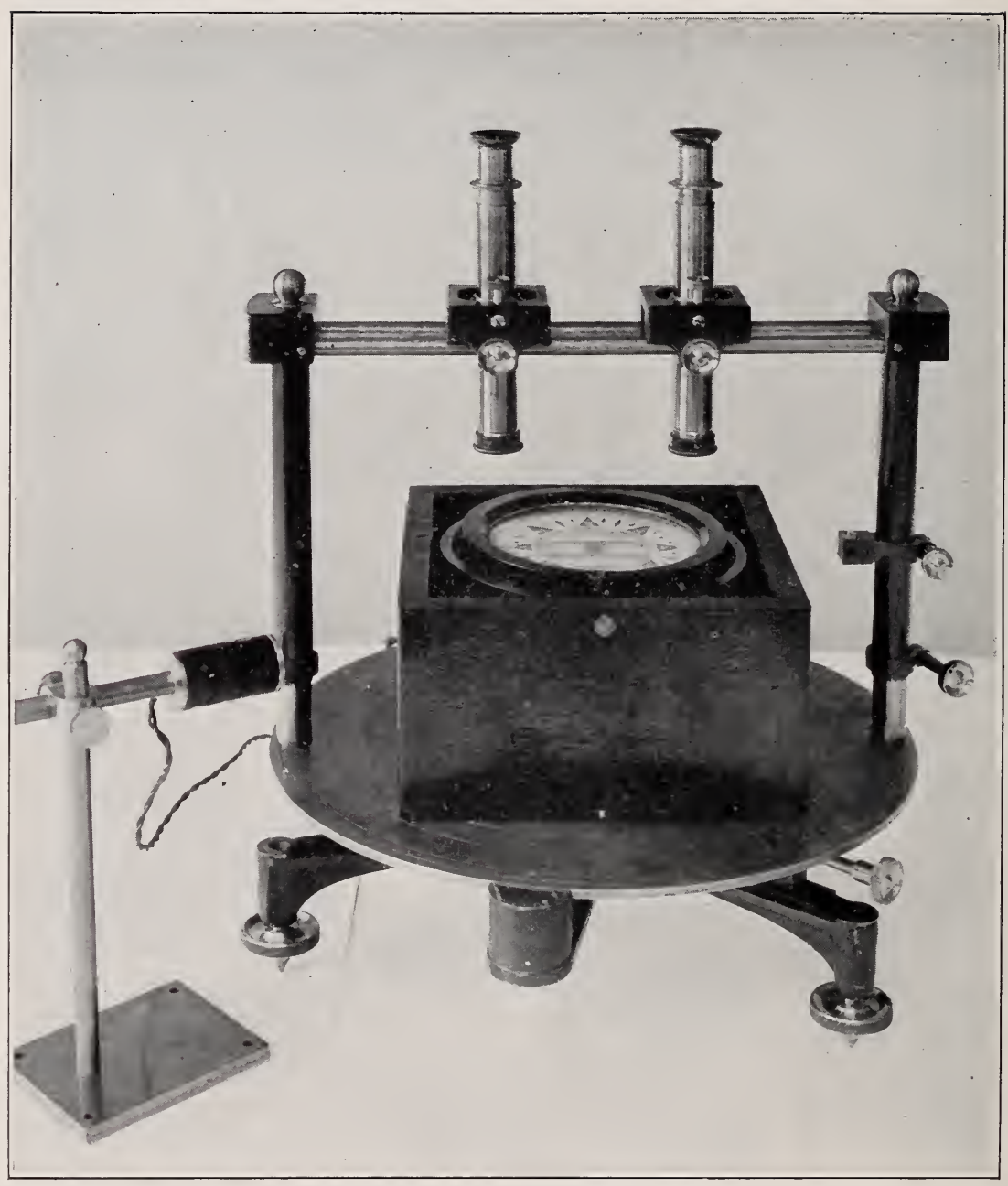

FIG. 2.-Testing stand 
a vertical axis, graduated around its circumference so that any angle through which it may be turned can be read by means of an adjustable index. By means of a vernier angles can be read to o. ${ }^{\circ}$. Mounted on uprights are two telescopes for sighting on the compass under test or on a standard compass. The small coil shown in the photograph is for deflecting the compass while testing pivot friction, period, and damping. When compasses having vertical cards are to be tested some modification of the sighting arrangement is necessary.

The standard compass is shown in the photograph of Fig. 3. It consists of a single needle mounted on a diamond pivot and having a sapphire cup. The friction of this mounting is so small as to be entirely negligible. A line drawn on the upper surface of the needle indicates the direction of its magnetic axis. This has been carefully adjusted and the adjustment can be checked at any time, as the needle is reversible and can be mounted either side up. The line on top lies in the vertical plane passing through the points at the ends of the needle.

The procedure in testing the calibration of a compass is as follows: First, the compass to be tested is placed on the stand and the telescopes focused on the north and south points of the card. The compass is then removed to a distance and the standard compass is placed on the stand and raised or lowered by means of an adjustable stand till the index line is in focus. The telescopes are not disturbed in any way after the initial adjustment. The table is oriented so that the image of the index line on the standard compass coincides with the cross hairs of the telescopes and the adjustable index set to zero and clamped. It is necessary, of course, that this test be carried out in a place free from magnetic disturbances which will cause a change in the direction or intensity of the magnetic field.

When the table index has been adjusted by means of the standard compass the instrument to be tested is replaced on the stand and the error on the north heading determined by noting the difference between the compass reading and the index reading. The table is then rotated through definite angles, and the calibration error for any heading, which is the difference between the reading of the stand and the reading of the compass, is found. It is advisable after a test to replace the standard compass on the stand and check the index adjustment, in order to make sure that the direction of the magnetic meridian has not changed during the test. 
Scientific Papers of the Bureau of Standards, Vol. 16

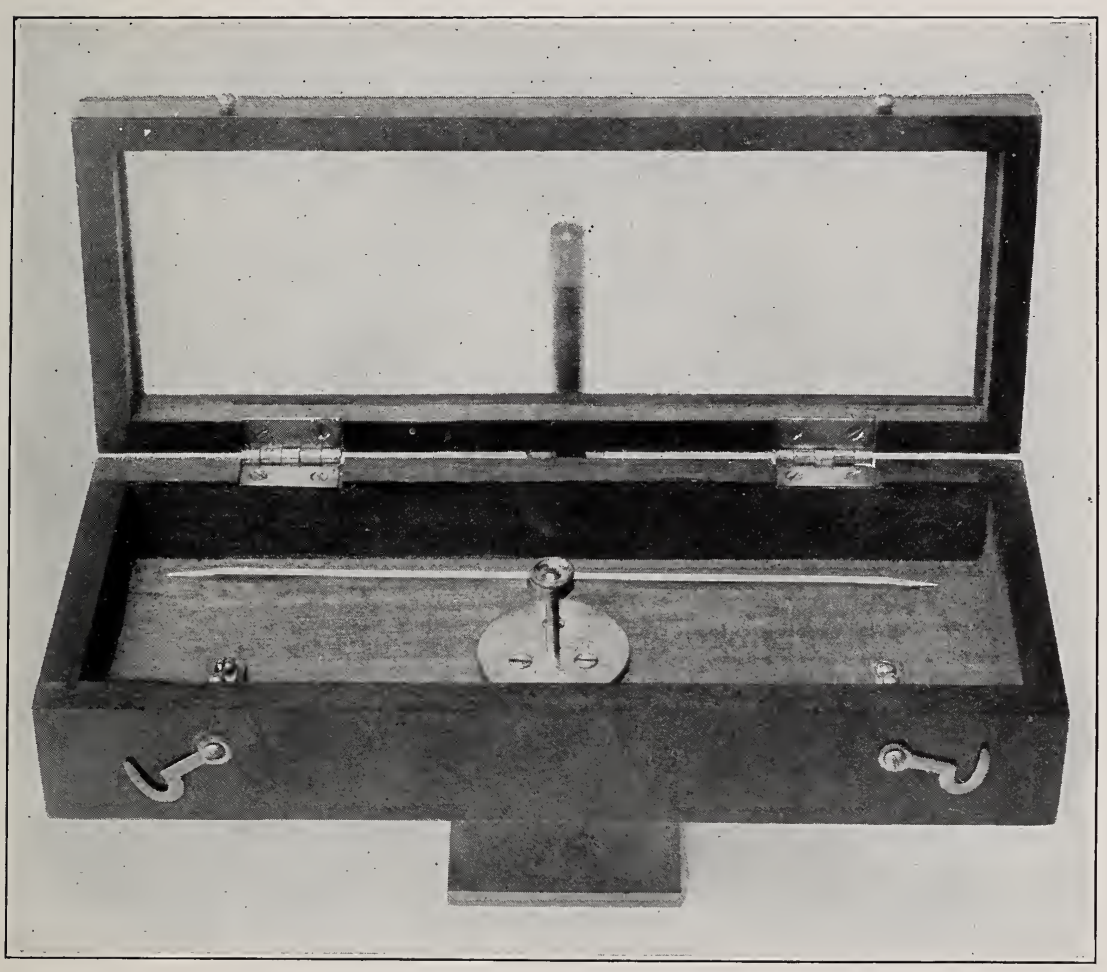

FIG. 3.-Standard compass 
3. PERIod.-The period or time of oscillation of a compass depends upon the moment of inertia of the card, the magnetic moment of the needles, and the horizontal intensity of the magnetic field. All other things being equal then, the period is a good indication of the strength of the magnetic needles. In some cases it is the custom to consider as the period the time which elapses between the instant that a swinging card passes through its position of equilibrium and the instant that it next passes through its equilibrium position on the return swing. Physically considered, this is only half a cycle, and so the time measured is the half period. In view of this fact it is necessary to make clear in specifications and reports of tests whether the complete or half period is used. The test is generally made by means of a stop watch and auxiliary magnet or coil to start the compass swinging. The card is set swinging and the time noted either for the half period or the complete period, according to the specifications. Any great variation from the normal period for the type of compass being tested is in general an indication of a variation in the strength of the magnetic needles.

One point should be borne in mind in measuring the period of a compass, namely, that the period depends not only on the strength of the needles but also upon the horizontal intensity of the magnetic field in which they swing. The normal variations in the earth's field are such that a compass having a period of 20 seconds in Washington, for instance, would have a period of 23 seconds in Bangor, Me., and 7 seconds in New Orleans.

4. DAmping.- In most marine compasses and in some other types some kind of liquid is employed. This liquid has the double function of taking some of the weight from the pivot and of bringing the card to rest more quickly, thus making it possible to take a reading sooner after a disturbance than could otherwise be done.

The method of determining the damping of a compass is to find the ratio of two successive swings on the same side of equilibrium. In a system practically free from solid friction this ratio is constant and its logarithm is called the logarithmic decrement. In a compass, however, this ideal condition is not realized, and the ratio is not constant for various amplitudes of swing. It is necessary, therefore, in order to obtain comparable results that the readings always be taken with approximately the same initial deflection. It is not proper to take the observation for damping by releasing the card from the required initial deflection. The system must be swinging freely. 
5. MAGNETIC Moment.-It is generally not necessary to make a direct measurement of the magnetic moment of the needle system, but it is desirable in some cases. For this purpose a special magnetometer (shown in Fig. 4) has been constructed. "The suspended system carries besides the needle a concave mirror and an aluminum vane which swings in an inclosed chamber and serves to damp the oscillations and bring the system quickly to rest. A permanent magnet is mounted on the instrument. This magnet is adjustable in position, and by its use the orientation and sensitivity of the suspended needle can be controlled. When the control magnet is so placed that the suspended needle takes up an east-west position, it is possible to determine the magnetic moment of a compass to a fair degree of accuracy (about 5 per cent) without arresting the compass card, which is a difficult matter in most liquid-filled compasses. The instrument is mounted so that the suspended needle is at the center of a large coil of known constants which is used for calibration. A support, also shown in the photograph, has been constructed for holding permanent bar magnets such as are used for compensating or adjusting compasses. It is prefereable to carry out this kind of work in a place very free from magnetic disturbances. It is because this condition is not realized at the Bureau that higher accuracy than 5 per cent is not attained. Fortunately, however, this degree of accuracy is satisfactory for most of the work with compasses.

\section{SUGGESTED PERFORMANCE SPECIFICATIONS}

The following suggested performance specifications cover the points just considered. The numerical values represent reasonable limits for a certain type of airplane compass. With suitable modifications of the requirements these specifications could be used to cover the performance of other types.

I. Pivor Friction.-When released from an initial deflection of $5^{\circ}$ in either direction the card shall in all cases return to its original position of rest within $\mathrm{I}^{\circ}$. This test to be repeated with the lubber line (or other sighting device) set on each of the cardinal points of the compass.

2. Calibration.-The calibration error in any direction shall not exceed $2^{\circ}$. The calibration error is the difference between the reading of the compass and the correct reading on any heading.

3. PERIOD.-The complete period, in a place where the horizontal intensity of the earth's magnetic field is 0.20 , shall not 
Scientific Papers of the Bureau of Standards, Vol. 16

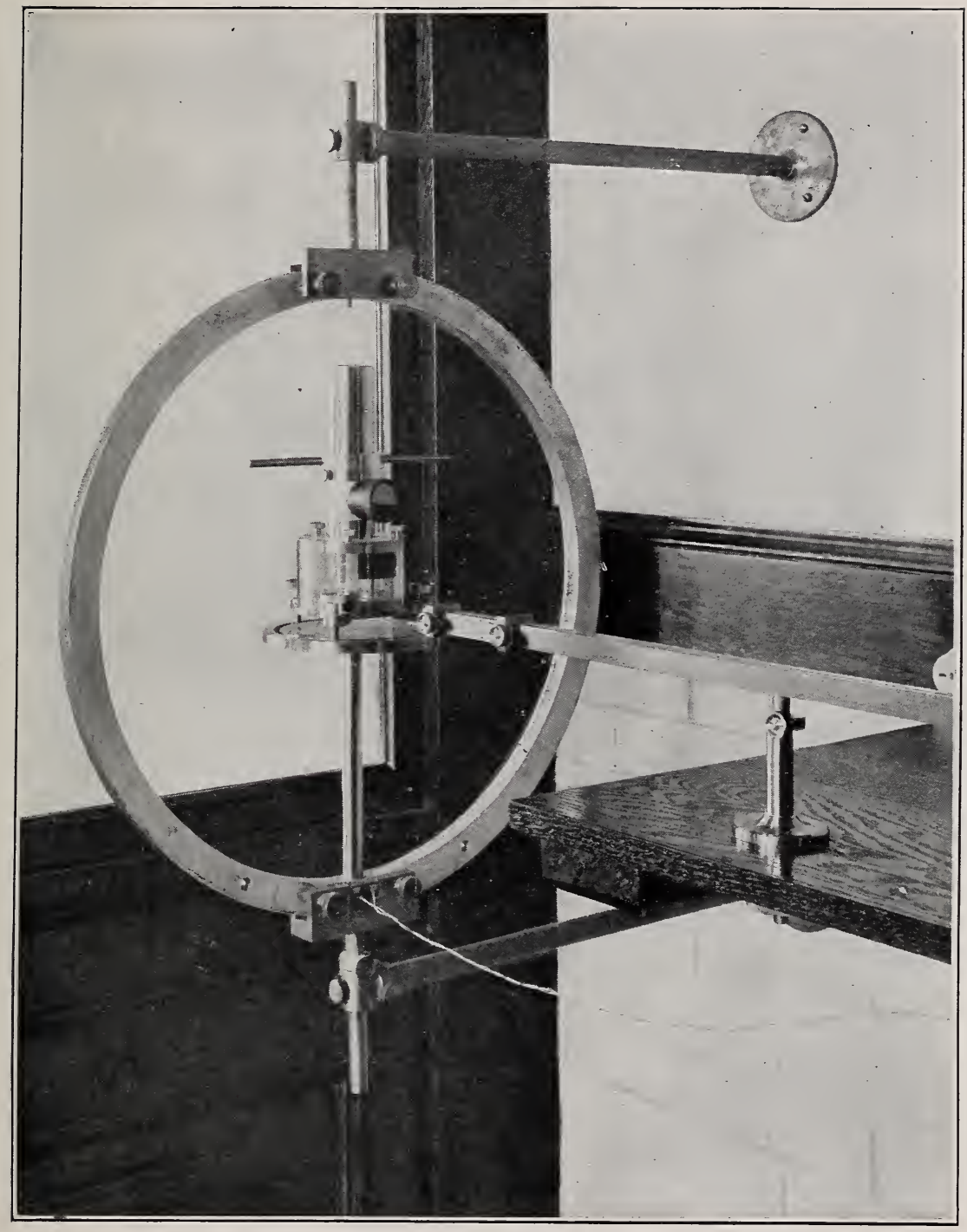

FIG. 4.-Magnetometer 
exceed 15 seconds. The period shall be taken as twice the time which elapses between successive transits of the card in opposite directions through its position of equilibrium when released from an initial deflection of $45^{\circ} .^{2}$

4. DAMPIng.-The damping constant determined from an initial swing of $45^{\circ}$ shall not be less than 15 nor greater than 45 . The damping constant is taken as the ratio of consecutive deflections on the same side of equilibrium when the card is swinging.

5. MAGNETIC Moment.-The magnetic moment of the needles shall be not less than $90 \mathrm{cgs}$ units. This determination need not be made unless the period exceeds 15 seconds.

\section{SUMMARY}

The work of the Bureau of Standards on magnetic compasses was undertaken in response to requests for information and cooperation from the War Department and the U. S. Shipping Board. It consisted of studies of the behavior of various types of compasses, the construction of special apparatus for testing purposes, and special investigations. The information thus obtained was utilized in the preparation of specifications for various types of compasses and also in the preparation of specifications for testing.

In the course of this investigation certain facts concerning the general characteristics of compasses were brought out which were considered to be of interest to users of magnetic compasses. This paper gives a brief discussion of the principal performance characteristics of magnetic compasses, together with a description of some of the apparatus which is used at the Bureau for testing.

WASHington, December Io, I920.

${ }^{2}$ In some types where the damping is not so great it is possible to measure the complete period. 





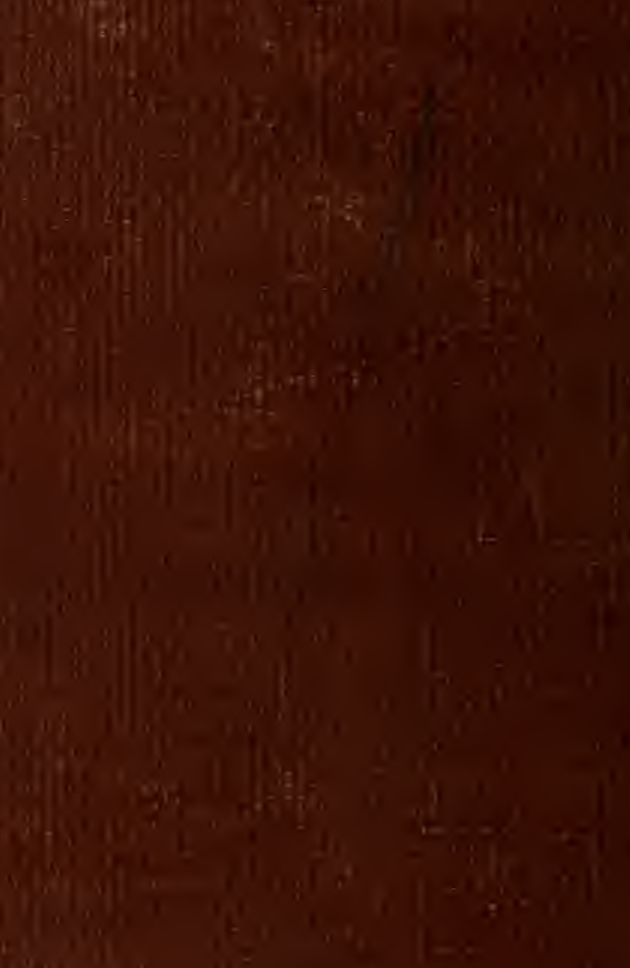

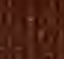
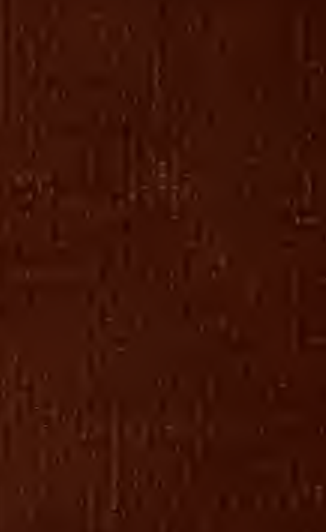

now 